\title{
A High-Resolution Laser Beam Collimator System with a High-NA Lens for a High-Density Ternary Barcode Detection System
}

\author{
Hiroo Wakaumi \\ Division of Electronics and Information Engineering, Tokyo Metropolitan College of Industrial \\ Technology, Japan \\ wakaumi@s.metro-cit.ac.jp
}

\begin{abstract}
A laser beam collimator system with a high numerical aperture lens and a $1.2 \mathrm{~mm}$ aperture masked collimator is proposed to realize a miniature high-resolution ternary barcode detection system in which high-density ternary barcodes can be detected. It was clarified that a $1.2 \mathrm{~mm}$ aperture mask combined with a $0.5 \mathrm{NA}$ lens is suitable for maintaining the laser beam width at less than $150 \mu \mathrm{m}$ over a wide range of more than $4.5 \mathrm{~cm}$. The laser head with this collimator system was applied to a ternary barcode detection system. It was confirmed that the detection system could detect the ternary barcode with a minimum bar width of $0.2 \mathrm{~mm}$ over a practical detection range greater than $5 \mathrm{~cm}$ (which conventional detection systems could not detect) by optimizing the clamp bias voltage in the clamping circuit to $-0.8 \mathrm{~V}$, and the gray bar concentration to $58 \%$. Barcode detection results also showed that an optimized mask aperture for achieving a maximum detection range for the barcode with a $0.2 \mathrm{~mm}$ minimum bar width was $1.2 \mathrm{~mm}$. This collimator system has potential applications in high-speed, high-resolution ternary or binary barcode detection systems.
\end{abstract}

Keywords: Optical detection system; Laser diode; Barcode; Ternary; Collimator.

\section{Introduction}

Real-time identification of barcodes is needed for applications such as goods management on production lines where high-speed detection is required. Though identification systems for mono or color two-dimensional binary barcodes using a CCD camera have been considered, its scanning speed is limited to nearly 50 scans/sec because of the complicated image processing and the need for focus adjustment [1,2]. This makes the high-speed sorting of goods problematic. In addition, an auxiliary light must be provided.

The authors have developed ternary barcode detection systems (BCDSs) using laser diode 
(LD) scanning to resolve the above problems of low-speed scanning, focus adjustment and auxiliary lighting requirements [3-5]. In particular, the ternary BCDS using the dual-bias differential method could detect a $0.25 \mathrm{~mm}$ wide ternary barcode within a $7.4 \mathrm{~cm}$ detection range. This performance has also been achieved with long detection distances of around $35 \mathrm{~cm}$ [5]. However, the detection of a minimum $0.2 \mathrm{~mm}$ wide ternary barcode equivalent to conventional binary barcodes has not yet been achieved. In conventional ternary BCDSs, a low numerical aperture $(N A=0.26)$ non-spherical lens with a relatively wide aperture $(2.5-3 \mathrm{~mm})$ optical masked collimator (consisting of a thin vinyl chloride sheet mask with a central hole), has been considered for achieving a narrow laser beam over a wide range for realizing as high a detection resolution as possible [6]. This is because a slightly wider aperture mask fabricated with high accuracy can be easily used because of an intrinsically wide depth of focus of the lens itself. However, it resulted in an insufficient beam width for detection distances over $35 \mathrm{~cm}$ while it could achieve a uniform beam over a wide range. Because of this inadequate performance of the optical collimator system in collimating the LD light, the detection resolution for the ternary $B C D S$ is limited to $0.25 \mathrm{~mm}$. Therefore, the development of a highresolution optical collimator system that provides a narrower laser beam over a wide range, is required to increase the detection resolution. As an approach to increasing the detection resolution, the use of a combination of a non-spherical lens and beam expander was considered. While the beam expander is useful for extending the width of the laser light beam before condensing it with the focusing non-spherical lens to achieve a narrow beam $[7,8]$, this approach results in a large and expensive system. Because a miniature BCDS is desirable for practical applications, it is also essential to fabricate the system compactly.

In this study, a high-resolution laser beam collimator system consisting of a high NA nonspherical lens providing high resolution and a narrow aperture masked collimator operating as a narrow optical stop for a laser light beam [9], is proposed. This is to provide a high quality laser beam and a wide depth of focus for short detection distances, resulting in the achievement of a small high-density ternary BCDS.

\section{High Resolution Collimator}

The conventional collimator system, using a low NA (NA=0.26) non-spherical lens with an intrinsic focal length of $14.5 \mathrm{~mm}$, is required to adjust a masked collimator aperture size for a scanning distance of over $35 \mathrm{~cm}$ when it is desirable to achieve a uniform laser beam over a wide range. When a masked collimator with a hole diameter (aperture) $\Phi$ of $2.5 \mathrm{~mm}$ was attached after adjusting the focal distance of the lens to near $35 \mathrm{~cm}$, the laser beam widths became 175-185 $\mu \mathrm{m}$ for a scanning range of $5 \mathrm{~cm}$ as shown in Fig. 1 [6]. These laser beam widths were not narrow enough to detect the $0.2 \mathrm{~mm}$ wide high-density ternary barcode and so limited the barcode detection resolution to $0.25 \mathrm{~mm}$.

Resolution of lens $\delta$ is given by K/NA [10]. Here, $\mathrm{K}$ is constant. Considering this fact, the 
increase of NA of the lens is needed to increase the detection resolution. Narrowing a mask aperture of masked collimator is also needed to achieve more uniform beam over a wide range. Such a high resolution collimator system can keep a narrow beam within a wide range as shown in Fig. 2. Based on such ideas, the proposed collimator system uses a high resolution nonspherical lens with an NA of 0.5 and a narrow-aperture masked collimator, to realize a short focus laser head corresponding to short distance scanning. The position of the high 0.5 NA nonspherical lens with an intrinsic focal length of $4 \mathrm{~mm}$ was designed to provide a focal distance of $15 \mathrm{~cm}$. The masked collimator with an aperture of $\Phi=1.2-1.5 \mathrm{~mm}$ was attached to the laser head to retain a uniform laser beam over a wide range of nearly $5 \mathrm{~cm}$.

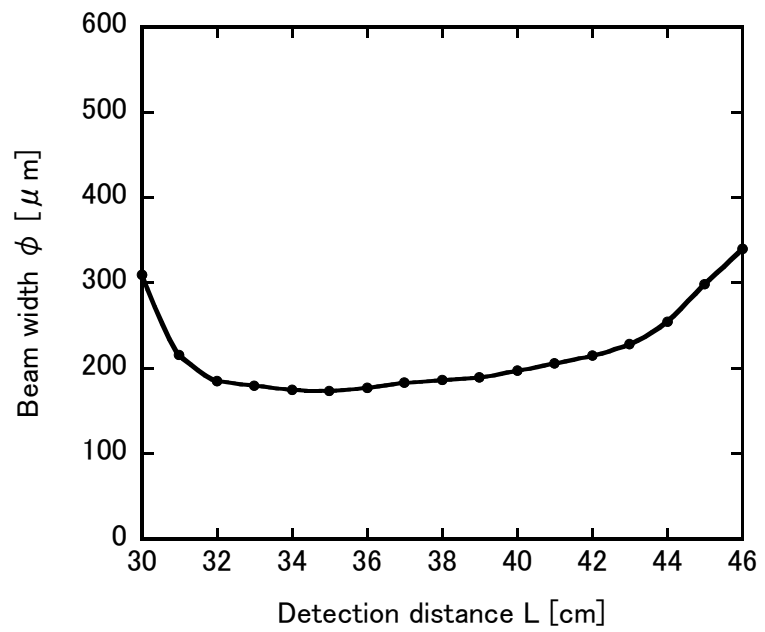

Figure 1: Beam width versus detection distance in a conventional collimator laser head. $\Phi=2.5 \mathrm{~mm}$

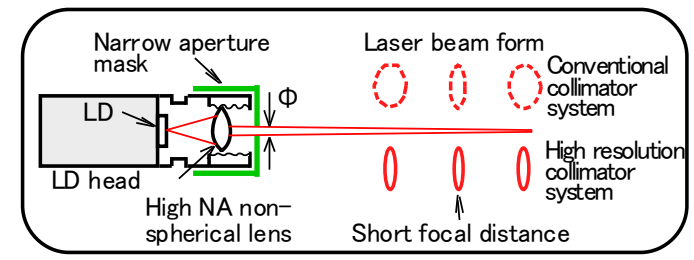

Figure 2: Laser beam forms for conventional collimator and high-resolution collimator systems

\section{Experimental Results}

\subsection{Laser Beam Width}

The laser head using the conventional low-NA non-spherical lens cannot simply shorten its focal distance. As described above, because the beam width of the laser light emitted from the laser head was 175-185 $\mu \mathrm{m}$ around a focal distance of nearly $35 \mathrm{~cm}$, it was impossible to apply it to high density ternary BCDSs. Even if its focal distance was shortened to $15 \mathrm{~cm}$ by adjusting the position of a lens attached in front of the laser equivalent to a small scanner, the scanning range of a laser head with a masked collimator of $\Phi=1.2 \mathrm{~mm}$ producing light beams narrower than $150 \mu \mathrm{m}$ was below $2 \mathrm{~cm}$, which was extremely narrow (Fig. 3). This was near the limitation of the laser beam width required for detecting $0.2 \mathrm{~mm}$ wide ternary barcodes. 


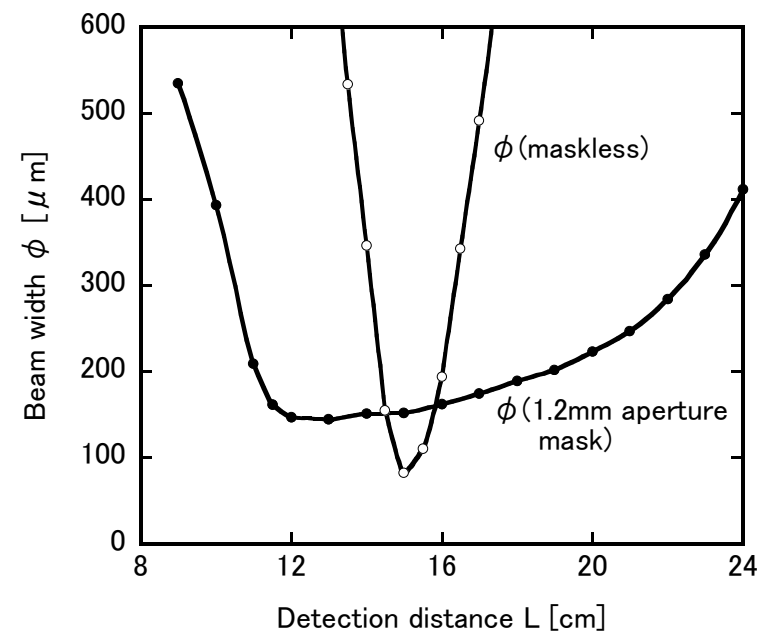

Figure 3: Beam width versus detection distance when the focal distance is adjusted to $15 \mathrm{~cm}$ using a conventional laser head

The laser head using the proposed collimator system consisted of a semiconductor laser of DL3149-057 (lasing wavelength $\lambda=670 \mathrm{~nm}$ ) with a non-spherical lens of NA=0.5 and an intrinsic focal length of $4 \mathrm{~mm}$ as described above. The beam width versus detection distance characteristics with masked collimators of $\Phi=1.2 \mathrm{~mm}$ and $1.5 \mathrm{~mm}$ (including that without a masked collimator) are shown in Fig. 4. Although, without a masked collimator, the beam width at a detection distance $L=15 \mathrm{~cm}$ decreased to $70 \mu \mathrm{m}$ resulting from the increase in $N A$, the scanning range $\Delta \mathrm{L}$ producing light beams narrower than $150 \mu \mathrm{m}$ was $2 \mathrm{~cm}$, which was still too narrow. Conversely, when a laser head using masked collimators with apertures of $1.2 \mathrm{~mm}$ and $1.5 \mathrm{~mm}$ was used, the scanning range extended to $4.5 \mathrm{~cm}$ and $4.4 \mathrm{~cm}$, respectively. Thus, it was decided that a high-resolution laser beam collimator system consisting of a high NA nonspherical lens with a $1.2 \mathrm{~mm}$ wide masked collimator was the most useful for realizing miniature BCDSs with wide scanning ranges.

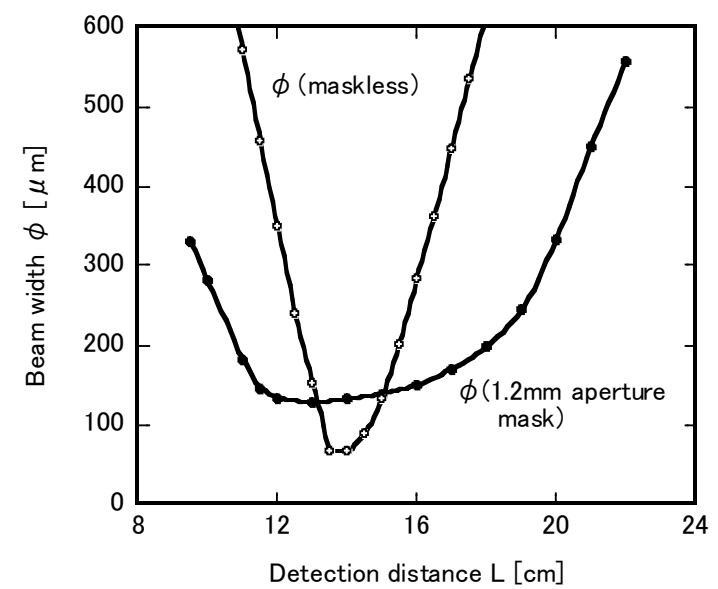

(a)

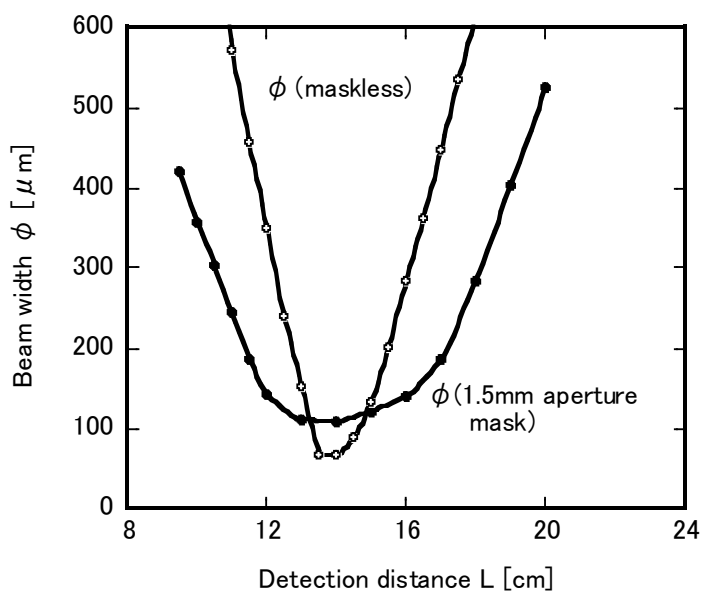

(b)

Figure 4: Beam width versus detection distance when the masked collimator aperture is $1.2 \mathrm{~mm}(\mathrm{a})$ and $1.5 \mathrm{~mm}(\mathrm{~b})$ in the proposed high NA system 


\subsection{Application to Ternary BCDS}

The laser head combined a high NA lens with a masked collimator of $\Phi=1.2 \mathrm{~mm}$. This was expected to realize the widest scanning range, and was applied to a ternary BCDS including a four-character ternary barcode (consisting of nine elements per character except for start and stop codes) as shown in Fig. 5. The detection characteristics were measured. The distance between the polygonal rotating mirror and the laser head was $7 \mathrm{~cm}$. The scanning distance indicating the distance between the laser head and the surface of the barcode was nearly $15 \mathrm{~cm}$ on average. In this system, the detection range was controlled by adjusting the average level of gray signals using a clamp bias voltage in the clamping circuit [11]. It was expected that the average level of the gray signals changed depending on barcode pattern density and gray bar concentration. Figure 6 shows detection signal processing waveforms depending on a gray bar concentration. Actually, it is seen that gray signals in a clamped average signal increase as the gray bar concentration increases from $52 \%$ to $58 \%$. This fact indicates the need of optimization of the clamp bias voltage because differential signals change depending on an average level of the clamped average signal. Figure 7 shows a change of the occurrence of code errors depending on the clamp bias voltage. Strong saturation in differential signals was seen without a clamp bias, causing code errors in a decodable signal. However, when the clamp bias voltage of $-0.8 \mathrm{~V}$ was applied, differential signals came not to saturate strongly. This weak saturation caused correct codes in the decodable signal. Consequently, applying some clamp bias voltages seems desirable.

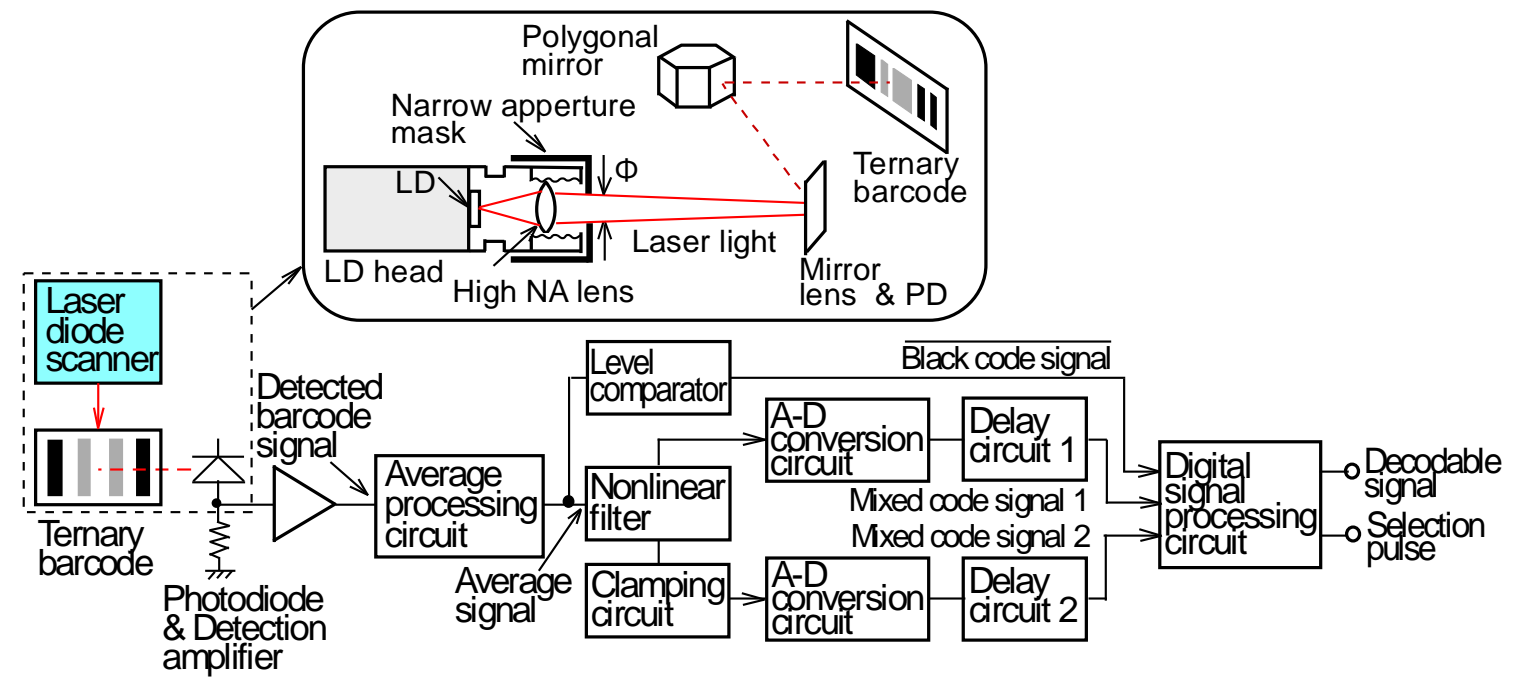

Figure 5: A ternary barcode detection system configuration 


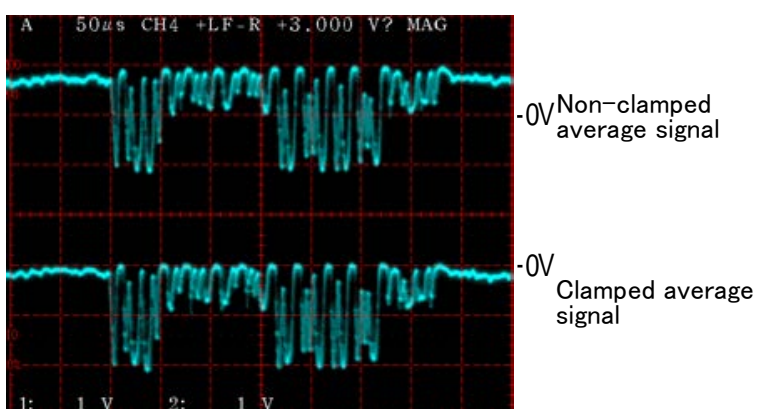

(a)

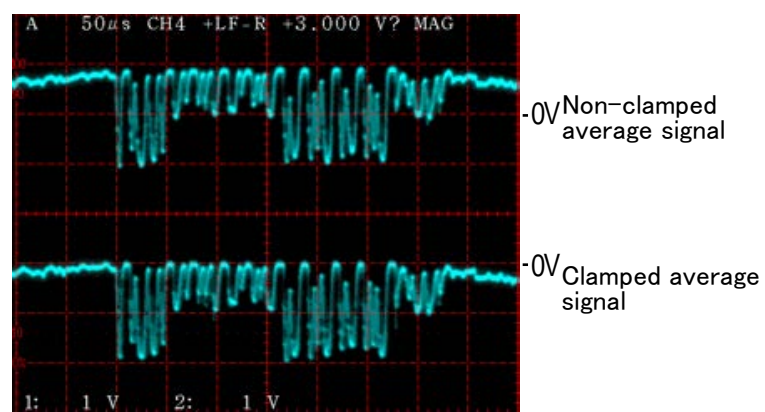

(b)

Figure 6: Detection signal processing waveforms depending on a gray bar concentration of $52 \%$ (a) and 58 \% (b)

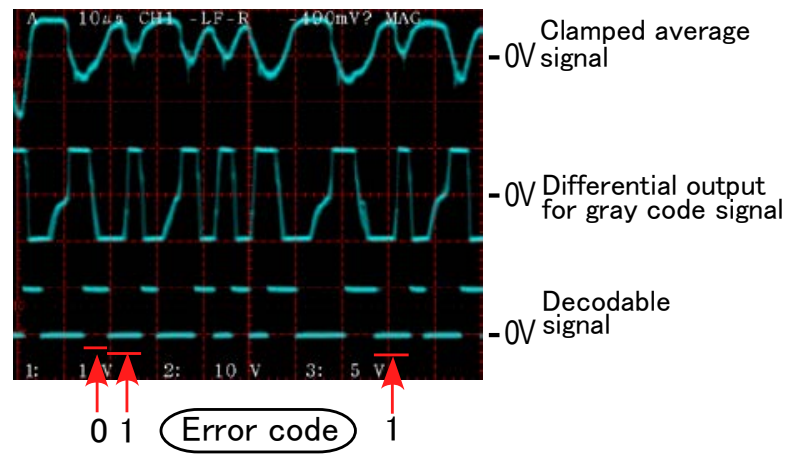

(a)

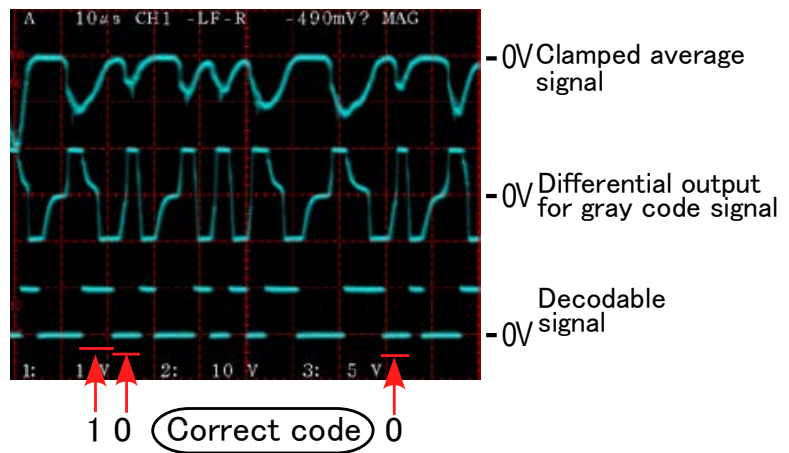

(b)

Figure 7: Detection signal processing waveforms depending on a clamped bias voltage $V_{\text {bias }}$ of $0 \mathrm{~V}$ (a) and -0.8 $\mathrm{V}$ (b)

Thus, based on these facts, the detection characteristics for the clamp bias voltage with respect to different gray bar concentrations when using the barcode with a minimum bar width of $W=0.2 \mathrm{~mm}$, were compared (Fig. 8(a) and (b)). It is apparent that when the gray bar concentration is increased from the conventional value of $52 \%$ to $58 \%$, compared with that of the white bar, the detection range is greatly extended for a clamp bias voltage $V_{\text {bias }}$ of near $-0.8 \mathrm{~V}$. Although the gray signals corresponding to narrow gray bars specifically diminish for a narrow ternary barcode of nearly $0.2 \mathrm{~mm}$, the increase in the detection range is thought to be because these gray signals increase (resulting in a wide detection range) when increasing the gray bar concentration. When this gray bar concentration is increased further, the detection range decreased at the same clamp bias voltage. 


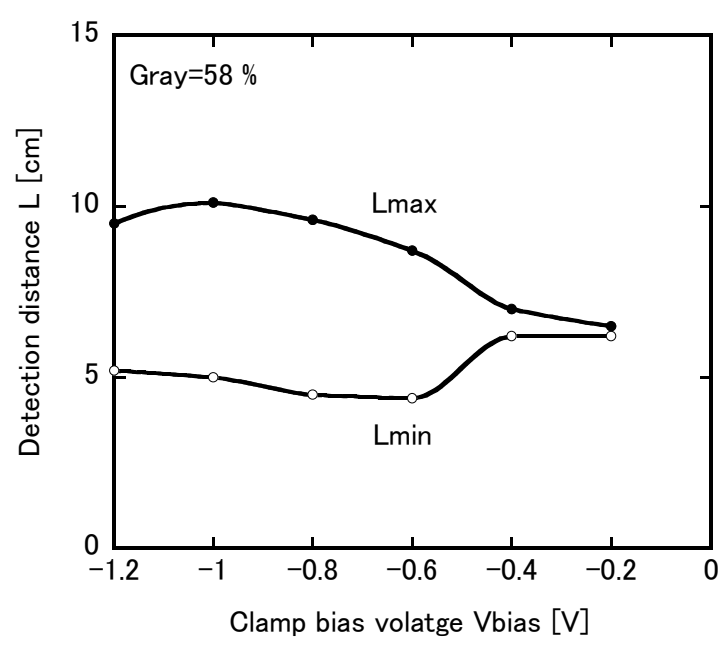

(a)

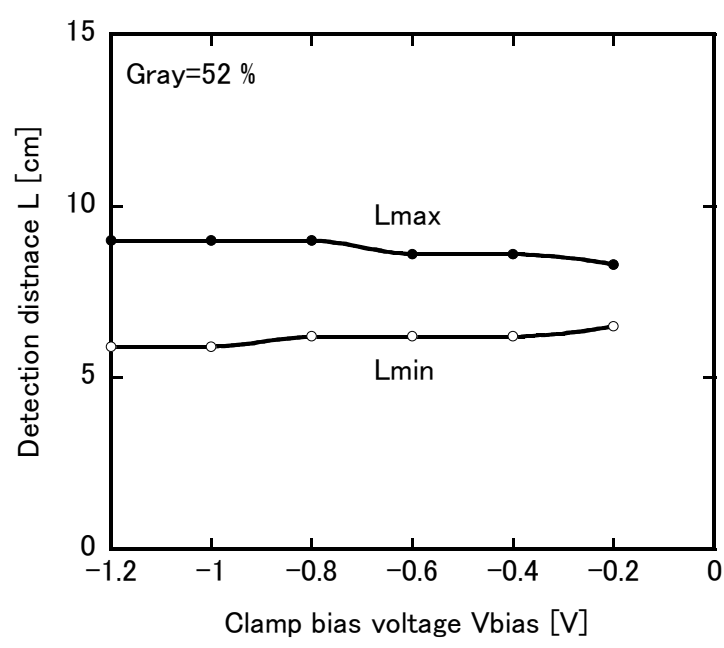

(b)

Figure 8: Detection distance versus clamp bias voltage with a gray bar concentration of $58 \%$ (a) and $52 \%$ (b). $\mathrm{W}=0.2 \mathrm{~mm}$. Scanning speed $\mathrm{fs}=333 \mathrm{scans} / \mathrm{sec}$. Light output power of the LD measured through the masked collimator of $\Phi=1.2 \mathrm{~mm}: P_{0}=0.6 \mathrm{~mW}$

This decrease in the detection range is also thought to be because gray signals resulting from a further increase in the gray bar concentration caused the system to exceed the comparator level of the black signals. At the optimum clamp bias voltage mentioned above, the detection distance versus minimum bar width is shown in Fig. 9. We can see that though the detection range for the barcode with a $\mathrm{W}$ of over $0.25 \mathrm{~mm}$ is not quite dependent on the gray bar concentration, a high gray bar concentration is effective in extending the detection range for a narrow barcode of $\mathrm{W}=0.2 \mathrm{~mm}$. In this regard, when the gray bar concentration was increased by $6 \%$ to $58 \%$, a practical detection range greater than $5 \mathrm{~cm}$ was obtained even for a high density barcode of $W=0.2 \mathrm{~mm}$. This could not be achieved in the conventional BCDS with a scanning distance of nearly $35 \mathrm{~cm}$.

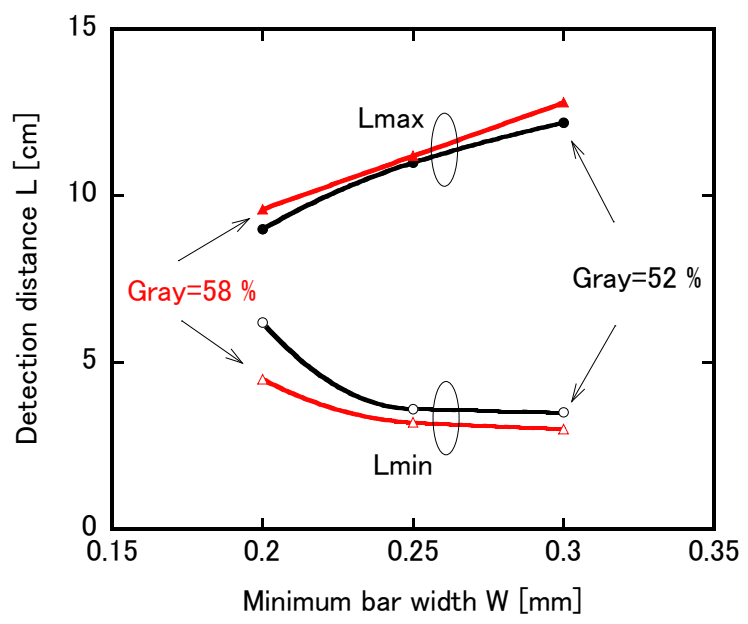

Figure 9: Detection distance versus minimum bar width depending on the gray bar concentration. fs=333 scans $/ \mathrm{sec}$. $V_{\text {bias }}=-0.8 \mathrm{~V} . \Phi=1.2 \mathrm{~mm} . P_{0}=0.6 \mathrm{~mW}$ 
Though a $1.2 \mathrm{~mm}$ aperture mask was chosen in section 3 from the high possibility of achievement of narrow beam width within a wide range, barcode detection characteristics were actually tested to confirm the optimum masked collimator aperture size. Figure 10 shows detection distance versus masked collimator aperture. It is apparently seen that a $1.2 \mathrm{~mm}$ mask aperture is most desirable for a wide detection range. When this mask was used, the detection range became maximum.

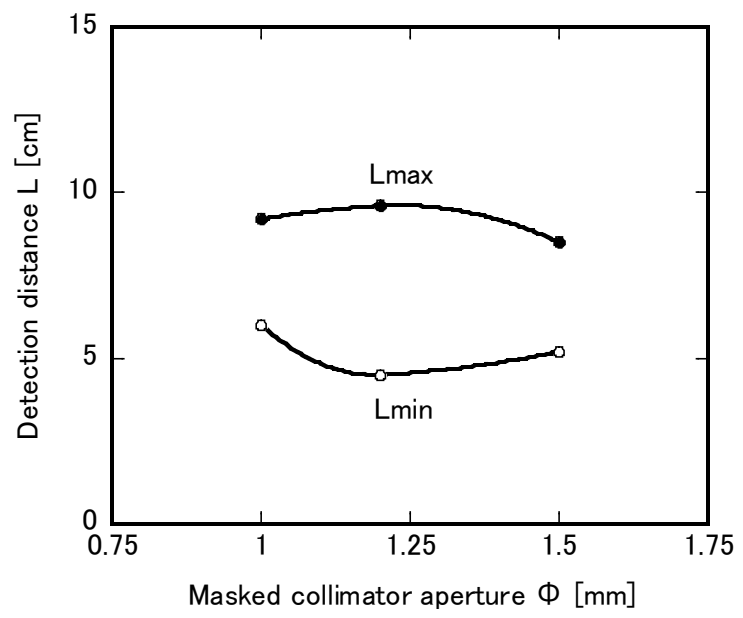

Figure 10: Detection distance versus masked collimator aperture. $V_{\text {bias }}=-0.8 \mathrm{~V}$

Thus, it was established that by applying a high 0.5 NA non-spherical lens with a narrow $1.2 \mathrm{~mm}$ aperture masked collimator, and optimizing the gray bar concentration and clamp bias voltage, a miniature ternary BCDS able to detect narrow $0.2 \mathrm{~mm}$ wide ternary barcodes, equivalent to conventional binary barcodes, could be achieved.

\section{Conclusions}

A high-resolution laser beam collimator system with a high numerical aperture nonspherical lens and a $1.2 \mathrm{~mm}$ aperture masked collimator has been proposed to realize a miniature ternary barcode detection system which can detect high density ternary barcodes. It was clarified that a $1.2 \mathrm{~mm}$ aperture mask combined with a $0.5 \mathrm{NA}$ lens is suitable for maintaining the laser beam width at less than 150 um over a wide range of more than $4.5 \mathrm{~cm}$. The laser head with this collimator system was applied to a ternary barcode detection system. As a result, it was confirmed that the detection system with the high-resolution collimator system could detect a ternary barcode with a minimum bar width of $0.2 \mathrm{~mm}$ for a practical wide detection range greater than $5 \mathrm{~cm}$, by optimizing the clamp bias voltage to $-0.8 \mathrm{~V}$ and the gray bar concentration to $58 \%$. Under this condition, an optimized mask aperture was $1.2 \mathrm{~mm}$ for achieving a maximum detection range for the barcode with a $0.2 \mathrm{~mm}$ minimum bar width. This collimator system has potential applications in high-speed, high-resolution ternary or binary barcode detection systems. 


\section{REFERENCES}

[1]. J. Hiramoto, Knowledge of Barcode and Two-Dimensional Code, $5^{\text {th }}$ Edition 2001, Tokyo: Japan Industrial Publishing (Japan).

[2]. T. Nagaya, T. Yamazaki, M. Hara, and T. Nojiri, Two-Dimensional Code for High-Speed Reading, in Proc. of the $52^{\text {th }}$ Information Processing Society of Japan (IPSJ) General Conference, 1996, pp. 253-254.

[3]. H. Wakaumi, An Envelope-Differential Composite Method for a High-Density Ternary Barcode Detection System, The IEICE Transactions on Fundamentals of Electronics, Communications and Computer Sciences (Japanese Edition), 2011, Vol. J94-A, No. 2, pp. 142-144.

[4]. H. Wakaumi, A High-Density Ternary Barcode Detection System Employing a Stable Fixed-Period Delay Method, Optical Review, 2011, Vol. 18, No. 5, pp. 408-413.

[5]. H. Wakaumi, A Ternary Barcode Detection System Employing a Dual-Bias Differential Method, in the $13^{\text {th }}$ Mechatronics Forum International Conference-Mechatronics 2012, 2012, pp. 399-403.

[6]. H. Wakaumi and C. Nagasawa, High detection resolution for two-dimensional bar-code detection system using masked collimators, Sensors and Actuators A: Physical, 2004, Vol. 110, pp. 177-181.

[7]. B. Grahame and T. Tsunehisa, Laser Diode Collimator System, Japan Patent Application number : 2003018131, Jan. 28, 2003.

[8]. T. Ohsawa and T. Obokata, Lasermetrics, $1^{\text {st }}$ Edition 1994, Tokyo: Shokabo (Japan).

[9]. H. Wakaumi, A High-Resolution Laser Beam Collimator System for a High-Density Ternary Barcode Detection System, in the $13^{\text {th }}$ International Conference on Control, Automation and Systems (ICCAS2013), 2013, pp. 1384-1387.

[10]. M. H. Freeman, Optics, $10^{\text {th }}$ Edition 1997, Oxford: Butterworth Heinemann (United Kingdom).

[11]. H. Wakaumi, A High-Density Ternary Barcode Detection System with a Dual-Bias Differential Method, Journal of Sensor Technology, 2013, Vol. 3, No. 1, pp. 6-12. 